\title{
Inter-reader agreement of the PI-QUAL score for prostate MRI quality in the NeuroSAFE PROOF trial
}

\author{
Francesco Giganti ${ }^{1,2}$ (D) Eoin Dinneen ${ }^{2,3} \cdot$ Veeru Kasivisvanathan $^{2,3} \cdot$ Aiman Haider $^{4} \cdot$ Alex Freeman $^{4} \cdot$ Alex Kirkham $^{1}$. \\ Shonit Punwani ${ }^{1,5} \cdot$ Mark Emberton $^{2,3} \cdot$ Greg Shaw $^{2,3} \cdot$ Caroline M. Moore $^{2,3} \cdot$ Clare Allen $^{1}$
}

Received: 9 April 2021 / Revised: 20 June 2021 / Accepted: 25 June 2021 / Published online: 29 July 2021

(C) The Author(s) 2021

\begin{abstract}
Objectives The Prostate Imaging Quality (PI-QUAL) score assesses the quality of multiparametric MRI (mpMRI). A score of 1 means all sequences are below the minimum standard of diagnostic quality, 3 implies that the scan is of sufficient diagnostic quality, and 5 means that all three sequences are of optimal diagnostic quality. We investigated the inter-reader reproducibility of the PI-QUAL score in patients enrolled in the NeuroSAFE PROOF trial.

Methods We analysed the scans of 103 patients on different MR systems and vendors from 12 different hospitals. Two dedicated radiologists highly experienced in prostate mpMRI independently assessed the PI-QUAL score for each scan. Interobserver agreement was assessed using Cohen's kappa with standard quadratic weighting $(\mathrm{KW})$ and percent agreement.

Results The agreement for each single PI-QUAL score was strong ( $\mathrm{KW}=0.85$ and percent agreement $=84 \%)$. A similar agreement $(\mathrm{KW}=0.82$ and percent agreement $=84 \%)$ was observed when the scans were clustered into three groups (PIQUAL 1-2 vs PI-QUAL 3 vs PI-QUAL 4-5). The agreement in terms of diagnostic quality for each single sequence was highest for T2-weighted imaging $(92 / 103$ scans; $89 \%)$, followed by dynamic contrast-enhanced sequences $(91 / 103 ; 88 \%)$ and diffusionweighted imaging $(80 / 103 ; 78 \%)$.

Conclusion We observed strong reproducibility in the assessment of PI-QUAL between two radiologists with high expertise in prostate mpMRI. At present, PI-QUAL offers clinicians the only available tool for evaluating and reporting the quality of prostate mpMRI in a systematic manner but further refinements of this scoring system are warranted.

Key Points

-Inter-reader agreement for each single Prostate Imaging Quality (PI-QUAL) score (i.e., PI-QUAL 1 to PI-QUAL 5) was strong, with weighted kappa $=0.85(95 \%$ confidence intervals: $0.51-1)$ and percent agreement $=84 \%$.

- Interobserver agreement was strong when the scans were clustered into three groups according to the ability (or not) to rule in and to rule out clinically significant prostate cancer (i.e., PI-QUAL 1-2 vs PI-QUAL 3 vs PI-QUAL 4-5), with weighted kappa $=0.82(95 \%$ confidence intervals: $0.68-0.96)$ and percent agreement $=84 \%$.

- T2-weighted acquisitions were the most compliant with the Prostate Imaging Reporting and Data System (PI-RADS) v. 2.0 technical recommendations and were the sequences of highest diagnostic quality for both readers in 95/103 (92\%) scans, followed by dynamic contrast enhanced acquisition with 81/103 (79\%) scans and lastly by diffusion-weighted imaging with 79/ $103(77 \%)$ scans.
\end{abstract}

Keywords Urogenital neoplasms $\cdot$ Prostatic neoplasms $\cdot$ Magnetic resonance imaging

Francesco Giganti

f.giganti@ucl.ac.uk

1 Department of Radiology, University College London Hospital NHS Foundation Trust, London, UK

2 Division of Surgery \& Interventional Science, University College London, 3rd Floor, Charles Bell House, 43-45 Foley St,

London W1W 7TS, UK
3 Department of Urology, University College London Hospital NHS Foundation Trust, London, UK

4 Department of Pathology, University College London Hospital NHS Foundation Trust, London, UK

5 Centre for Medical Imaging, University College London, London, UK 


$\begin{array}{ll}\text { Abbreviations } \\ \text { ADC } & \text { Apparent diffusion coefficient } \\ \text { DCE } & \text { Dynamic contrast enhanced } \\ \text { DWI } & \text { Diffusion-weighted imaging } \\ \text { mpMRI } & \text { Multiparametric magnetic resonance imaging } \\ \text { PACS } & \text { Picture Archiving and Communication System } \\ \text { PI-QUAL } & \text { Prostate Imaging Quality } \\ \text { PI-RADS } & \text { Prostate Imaging Reporting and Data System } \\ \text { PSA } & \text { Prostate-specific antigen } \\ \text { T2-WI } & \text { T2-weighted imaging }\end{array}$

\section{Introduction}

The development and subsequent diffusion of multiparametric magnetic resonance imaging (mpMRI) of the prostate have unavoidably resulted into variability in terms of vendors' and scanners' quality worldwide [1].

Suboptimal image acquisition reduces the diagnostic accuracy of mpMRI for the detection of clinically significant prostate cancer and this is why since the publication of the first version, the Prostate Imaging Reporting and Data System (PI-RADS) committee outlined the technical requirements for the acquisition of mpMRI of the prostate of adequate diagnostic quality [2].

Moreover, two boards of experts have reiterated the importance of quality criteria for the acquisition of mpMRI of the prostate $[3,4]$, and the Prostate Imaging Quality (PI-QUAL) scoring system [5] from the multi-centre PRECISION trial [6] has represented the first attempt to address this issue.

The PI-QUAL score evaluates the quality of prostate mpMRI against objective technical criteria (as per PI-RADS guidelines) together with subjective criteria from the images [5].

PI-QUAL is centred on a 1-to-5 scale that assesses the adequacy of the diagnostic quality of mpMRI of the prostate, where 1 implies that all mpMRI sequences are below the minimum standard of diagnostic quality, 3 means that the study is of sufficient diagnostic quality (as at least two mpMRI sequences taken together are of diagnostic quality), and 5 indicates that all sequences are of optimal diagnostic quality. In more detail, a PI-QUAL score $\geq 4$ implies that the quality of the study is high (i.e., all clinically significant lesions can be ruled in and ruled out).

The purpose of this study was to evaluate the inter-observer agreement of the PI-QUAL score in patients enrolled in the NeuroSAFE PROOF trial [7].

\section{Materials and methods}

This is a retrospective analysis of men prospectively enrolled in the NeuroSAFE PROOF trial (registration number: NCT03317990), whose recruitment started in 2018 [7]. The trial received ethical approval (Regional Ethics Committee
Fig. 1 The PI-QUAL scoring sheet used to assess the quality of multiparametric magnetic resonance imaging. Legend: T2-WI, T2weighted imaging; DWI, diffusion-weighted imaging; DCE, dynamic contrast-enhanced; ADC, apparent diffusion coefficient. Reprinted with permission from Giganti et al. [5]

reference 17/LO/1978) and was supported by the National Institute for Healthcare Research (NIHR reference PB-PG1216-20013). Written informed consent was obtained from all patients, who granted permission for their samples to be used for research purposes. No further ethical approval was needed for this specific audit.

The NeuroSAFE PROOF trial is an ongoing multicentre randomised controlled trial in which patients are randomised 1:1 to either NeuroSAFE (a technique that involves intraoperative fresh-frozen section analysis of the posterolateral aspect of the prostate margin to assess whether cancer extends beyond the capsule) or standard robot-assisted radical prostatectomy [7].

\section{Selection of patients}

Participants were recruited from different cancer centres routinely performing at least 250 cases of radical prostatectomy per year.

According to local protocols, all patients received a biparametric or multiparametric MRI study of the prostate prior to surgery on different MRI systems (Philips ${ }^{\circledR}$, Siemens ${ }^{\circledR}$, or General Electric () , on either a 1.5- or 3-T scanner, all without endorectal coil. During the trial, all scans were reported by dedicated genitourinary radiologists at each participating centre with at least 2 years of experience in prostate MRI reporting. At the same time, all scans were stored in a dedicated secured repository from which two designated radiologists (F.G. and C.A.) from the coordinating centre were able to download the scans for the assessment of image quality. As the PI-QUAL score [5] implies mpMRI of the prostate (i.e., including T2-weighted imaging (T2-WI), diffusionweighted imaging (DWI) and dynamic contrast-enhanced (DCE) sequences), we excluded those patients who received a biparametric scan.

\section{Assessment of image quality}

Two radiologists highly experienced in prostate MRI reporting (F.G., a fellowship-trained consultant radiologist reporting around 2000 prostate MRI scans per year and with 7 years of experience in prostate MRI, and CA, a senior consultant radiologist reporting more than 3000 prostate MRI scans per year and with 20 years of experience in prostate MRI) evaluated the image quality independently using the published PI-QUAL scoring sheet (Fig. 1). The adherence to technical parameters was tested against the PI-RADS v. 2.0 guidelines (as trial recruitment started in 2018, before the 
Scan \& site number:

0 PRECISION

Prostate Imaging QUALity control (PI-QUAL) scoring sheet

\begin{tabular}{|c|c|c|}
\hline PI-QUAL score & Criteria & Clinical implications \\
\hline 1 & All mpMRI sequences are below the minimum standard of diagnostic quality & \multirow{2}{*}{$\begin{array}{l}\text { It is NOT possible to rule in all significant lesions } \\
\text { It is NOT possible to rule out all significant lesions } \$\end{array}$} \\
\hline 2 & Only one mpMRI sequence is of acceptable diagnostic quality & \\
\hline$\overline{3}$ & At least two mpMRI sequences taken together are of diagnostic quality & $\begin{array}{l}\text { It is possible to rule in all significant lesions } \\
\text { It is NOT possible to rule out all significant lesions }\end{array}$ \\
\hline 4 & Two or more mpMRI sequences are independently of diagnostic quality & \multirow{2}{*}{$\begin{array}{l}\text { It is possible to rule in all significant lesions } \\
\text { It is possible to rule out all significant lesions }\end{array}$} \\
\hline 5 & All mpMRI sequences are of optimal diagnostic quality & \\
\hline
\end{tabular}

$\S$ Therefore reports should not include PI-RADS or Likert scores

Please $(\checkmark)$ if present: (note: 'adequote' means compliant with the technical specifications reported in PI-RADS $v .2$ guidelines) *

T2-WI

\section{Technical parameters}

Axial plane

Sagittal or coronal plane

Adequate field of view

Adequate in-plane resolution

Adequate slice thickness

Z-axis correctly positioned

\section{Visual assessment}

Capsule clearly delineated

Seminal vesicles clearly delineated

Ejaculatory ducts clearly delineated

Neurovascular bundles clearly delineated

Sphincter muscle clearly delineated

Absence of artefacts (e.g. movement)

Is T2-WI of diagnostic quality?
DWI

Technical parameters

Axial plane matching T2-WI

Adequate field of view

Adequate in-plane resolution

Adequate slice thickness

Multiple [ $>2$ ] $b$ values acquired

High $b$ value (synthesised or acquired)

\section{Visual assessment}

Adequate $A D C$ map

Absence of artefacts (e.g. rectal air)

Is DWI of diagnostic quality?

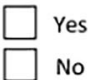

DCE

Technical parameters

Axial plane matching T2-WI

Adequate field of view

Adequate in-plane resolution

Adequate slice thickness

Pre-contrast T1-WI available

Fat suppression/subtraction

Adequate temporal resolution $[\leq 10 \mathrm{sec}]$

Adequate total observation rate [ $\geq 2 \mathrm{~min}]$

\section{Visual assessment}

Capsular vessels clearly delineated

Vessels in the Alcock's canal clearly delineated

Absence of artefacts (e.g. movement)

Is DCE of diagnostic quality?

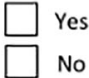

PI-QUAL score:

1

3

4

5

Date:

Reporting Radiologist:

Signed:

*Weinreb JC, et al. PI-RADS Prostate Imaging - Reporting and Data System: 2015, Version 2. Eur Urol 2016;69:16-40. 
publication of the PI-RADS v.2.1 guidelines) [8,9], as also stated in the original publication [5].

In detail, both readers assessed technical parameters such as field of view, in-plane resolution, and slice thickness for all MRI sequences and other specific parameters related to each single sequence (e.g. temporal resolution for DCE) and they visually assessed anatomical structures (e.g. the prostatic capsule on T2-WI or the pudendal vessels in the Alcock's - or pudendal - canal on DCE) using a dedicated Picture Archiving and Communication System (PACS) viewer (Vue PACS, Carestream Health, Inc).

Both readers were blinded to all clinical (including the original report and site key) and pathological information. All scans were anonymised and were read in randomised order by each reader.

It should be noted that although the two radiologists were blinded to the original report, for the purposes of this specific study they did not re-report the scans (i.e., no lesions were scored) but they deliberately focussed their work on the sole assessment of image quality.

\section{Pathologic analysis}

All patients received radical prostatectomy, either standard or using NeuroSAFE, at a single regional academic urooncology unit participating in the NeuroSAFE PROOF randomised controlled trial. The centre receives referrals from multiple centres in the vicinity as part of the National Health Service hub and spoke model for the referral of prostate cancer for surgery [7]. All pathological specimens were reported by dedicated urogenital pathologists.

\section{Statistical analysis}

Clinical and demographic data are reported using descriptive statistics. Continuous variables are summarised by median and interquartile ranges and categorical data by frequencies and percentages.

Inter-observer agreement was calculated using two methods: the percent agreement (defined as the total number of concordant readings divided by the total number of readings made) and Cohen's kappa with standard quadratic weighting (KW) using the formula: $\omega_{i}=1-\frac{i^{2}}{(k-1)^{2}}$, where $i$ is the difference between categories and $k$ is the total number of categories. Cohen's kappa coefficients (ranging from 0 to 1 ) were interpreted as follows: $0.01-020$, slight agreement; $0.21-0.40$, minimal agreement; $0.41-0.60$, moderate agreement; $0.61-0.80$, substantial agreement; $0.81-0.90$, strong agreement; and $>0.90$, almost perfect agreement [10-12].

Statistical analyses were performed using SPSS (IBM, version 27).

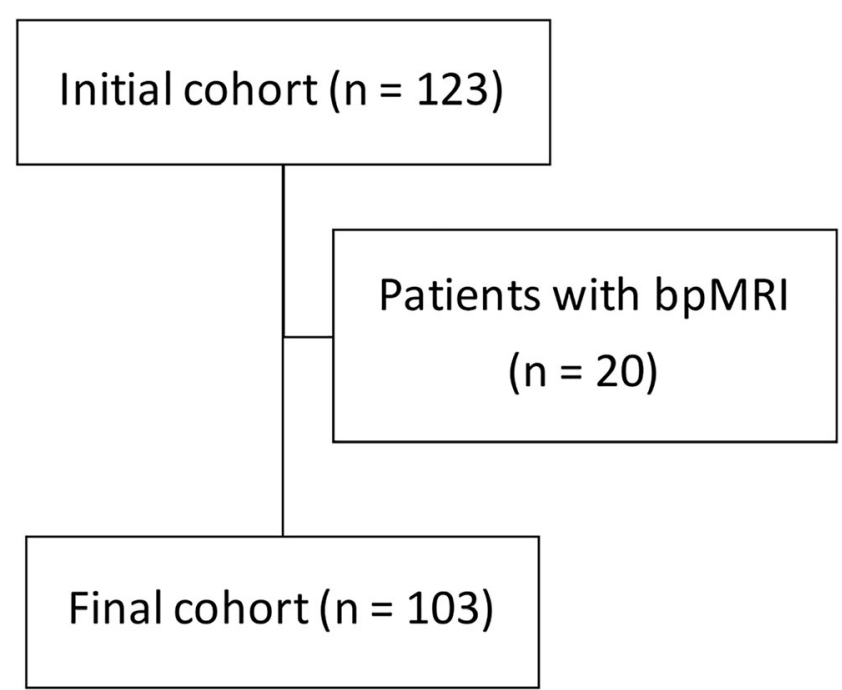

Fig. 2 Flowchart of the cohort included in this study. Legend: bpMRI, biparametric magnetic resonance imaging

\section{Results}

Among 123 patients from 13 centres who were enrolled in the study (as of February 2021), 20 (all from the same centre) had biparametric MRI, leaving 103 patients from 12 centres for final analysis (Fig. 2).

Patient characteristics are shown in Table 1.

Table 1 Characteristics of patients included in the study

\begin{tabular}{ll}
\hline & Population $(\mathrm{n}=103)$ \\
\hline Age (years) & $57[52-61]$ \\
PSA (ng/ml) & $6.9[5.1-9.4]$ \\
Prostate volume (cc) & $34[26-41]$ \\
PSA density $(\mathrm{ng} / \mathrm{ml} / \mathrm{ml})$ & $0.21[0.15-0.32]$ \\
Gleason Grade group at biopsy & \\
Gleason Grade 1 & $4(4 \%)$ \\
Gleason Grade 2 & $85(82 \%)$ \\
Gleason Grade 3 & $9(9 \%)$ \\
Gleason Grade 4 & $5(5 \%)$ \\
Gleason Grade group at radical prostatectomy & \\
Gleason Grade 1 & $1(1 \%)$ \\
Gleason Grade 2 & $84(81 \%)$ \\
Gleason Grade 3 & $16(16 \%)$ \\
Gleason Grade 4 & 0 \\
Gleason Grade 5 & $2(2 \%)$ \\
Pathological T stage at radical prostatectomy & \\
T2a & $3(3 \%)$ \\
T2b & $3(3 \%)$ \\
T2c & $66(64 \%)$ \\
T3a & $26(25 \%)$ \\
T3b & $5(5 \%)$ \\
\end{tabular}

Data are medians with interquartile ranges in brackets or number of patients with percentages in parentheses 
Overall, 83/103 (81\%) patients were scanned on a 1.5-T scanner and 20/103 (19\%) patients on a 3-T scanner, and the distribution in terms of MRI systems and manufacturers is shown in Fig. 3. The distribution of scans for each participating centre is reported in Table 2.

The main acquisition parameters for the different MRI scanners are reported in Supplementary Table 1.

\section{Reader agreement}

The agreement between readers for each single PI-QUAL score (i.e., PI-QUAL 1 to PI-QUAL 5) was strong, with $\mathrm{kW}$ $=0.85(95 \%$ confidence intervals: $0.51-1)$ and percent agreement $=84 \%$ (Table 3 ).

A strong agreement $(\mathrm{KW}=0.82$ [95\% confidence intervals: $0.68-0.96]$ and percent agreement $=84 \%$ ) was also observed when the scans were clustered into three groups according to the ability (or not) to rule in and to rule out clinically significant prostate cancer (i.e., PI-QUAL 1-2 vs PI-QUAL 3 vs PIQUAL 4-5) (Table 4).

The two readers showed disagreement in 16/103 (16\%) scans. In detail, 8/16 (50\%) scans were scored PI-QUAL 4 by reader 1 and PI-QUAL 3 by reader 2, 1/16 (6\%) scan was scored as PI-QUAL 3 by reader 1 and PI-QUAL 4 by reader 2, 4/16 (25\%) scans were scored PI-QUAL 3 by reader 1 and PI-QUAL 2 by reader 2, 2/16 (13\%) scans were scored as PI-QUAL 2 by
Table 2 Distribution of MR scans for each participating centre

\begin{tabular}{ll}
\hline & Number of MR scans \\
\hline Centre 1 & 31 \\
Centre 2 & 23 \\
Centre 3 & 10 \\
Centre 4 & 9 \\
Centre 5 & 8 \\
Centre 6 & 7 \\
Centre 7 & 6 \\
Centre 8 & 3 \\
Centre 9 & 2 \\
Centre 10 & 2 \\
Centre 11 & 1 \\
Centre 12 & 1 \\
Total & 103 \\
\hline
\end{tabular}

$M R$, magnetic resonance

reader 1 and PI-QUAL 3 by reader 2, and $1 / 16(6 \%)$ scan was scored as PI-QUAL 2 by reader 1 and PI-QUAL 4 by reader 2 .

As the PI-QUAL scoring sheet (Fig. 1) includes for each MRI sequence a two-step procedure that involves first the application of objective criteria for technical parameters (according to the PI-RADS v. 2.0 guidelines) and then a visual assessment of anatomical structures and image artefacts, we report the scores given by each reader for the 16 scans of disagreement in Table 5.

Fig. 3 MR manufacturers and systems included in the study

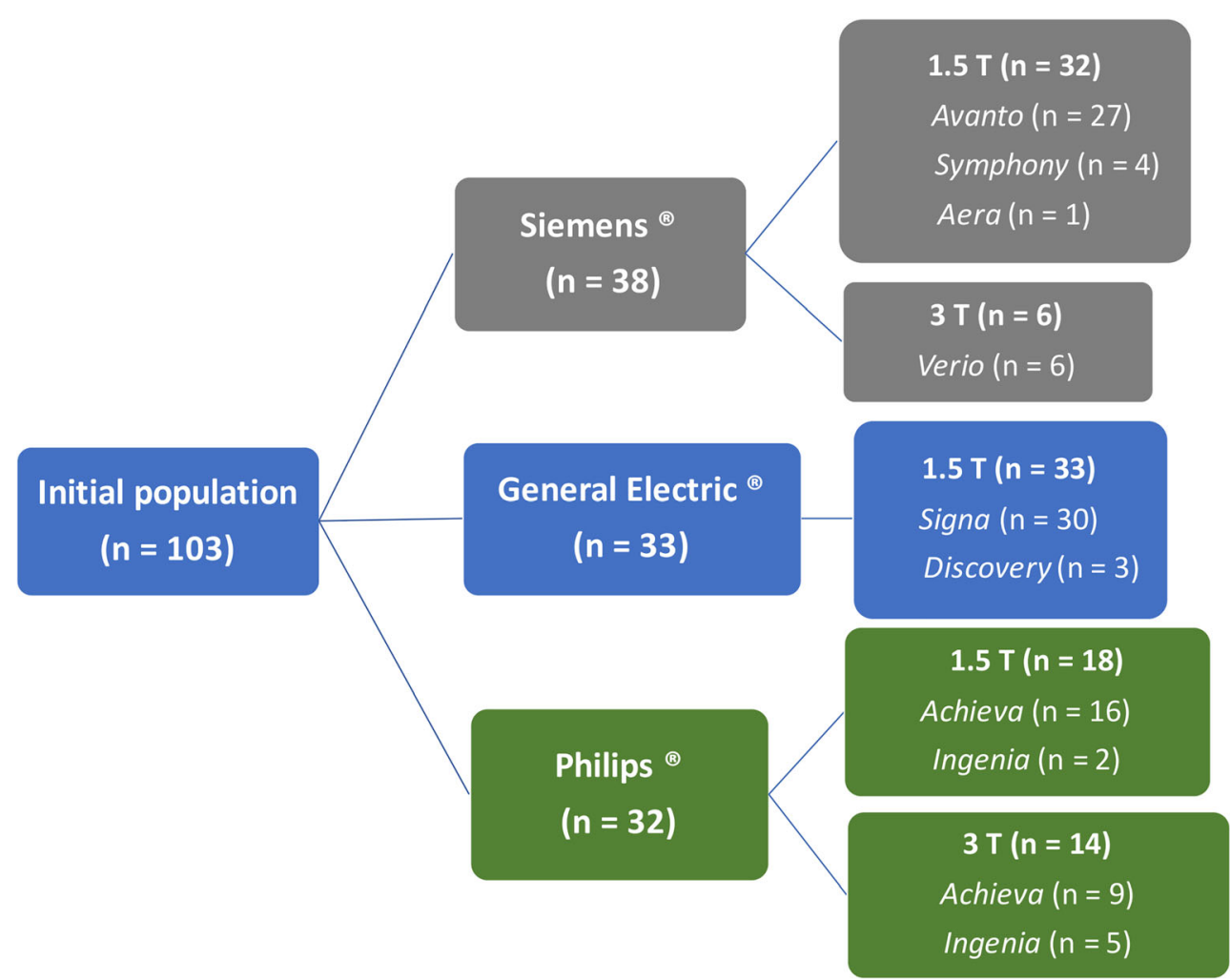


Table 3 Overall PI-QUAL scores $(n=103)$ as assessed by each reader

\begin{tabular}{llllllll}
\hline & \multicolumn{2}{l}{ Reader 2 } & & & & Total \\
\cline { 3 - 6 } & & PI-QUAL 1 & PI-QUAL 2 & PI-QUAL 3 & PI-QUAL 4 & PI-QUAL 5 & \\
\hline Reader 1 & PI-QUAL 1 & 1 & 0 & 0 & 0 & 0 & 1 \\
& PI-QUAL 2 & 0 & 14 & 2 & 1 & 0 & 17 \\
& PI-QUAL 3 & 0 & 4 & 38 & 1 & 0 & 43 \\
& PI-QUAL 4 & 0 & 0 & 8 & 32 & 0 & 40 \\
\multirow{2}{*}{ Total } & PI-QUAL 5 & 0 & 0 & 0 & 0 & 2 & 2 \\
\hline
\end{tabular}

PI-QUAL, Prostate Imaging Quality
After completion of the study, the two radiologists met and discussed the discordant cases for training and quality purpose. The final scores by consensus were as follows: $6 / 16$ (37.5\%) scans were scored as PI-QUAL 2, 4/16 (25\%) scans were scored as PI-QUAL 3 and 6/16 (37.5\%) scans were scored PI-QUAL 4 (Fig. 4).

As far as field strength is concerned (Supplementary Table 2), 65/83 (78\%) and 19/20 (95\%) scans had a PIQUAL score $\geq 3$ for 1.5-T and 3-T magnets, respectively. It should be noted that 3-T scanners represented only a small part $(19 \%)$ of the examinations included in this study.

As far as the diagnostic quality for each single sequence is concerned, the percent agreement between readers was highest for T2-WI (92/103 scans; $89 \%)$, followed by DCE sequences $(91 / 103 ; 88 \%)$ and lastly by DWI $(80 / 103 ; 78 \%)$.

In addition to this, T2-weighted acquisitions were the most compliant with the PI-RADS v. 2.0 technical recommendations across centres and were also the sequences of highest diagnostic quality for both readers in 95/103 (92\%) scans, followed by DCE acquisition with 81/103 (79\%) scans and lastly by DWI with 79/103 (77\%) scans (Figs. 5 and 6).

\section{Discussion}

In our study, we observed strong reproducibility in the assessment of prostate MRI quality using PI-QUAL between two radiologists with high expertise in prostate MRI. The agreement was strong both for each single PI-QUAL score ( $\mathrm{KW}=$ 0.85 and percent agreement $=84 \%)$ and when the PI-QUAL scores were clustered into three groups according to the ability (or not) to rule in and to rule out clinically significant prostate cancer $(\mathrm{KW}=0.82$ and percent agreement $=84 \%)$.

There is currently much interest in standardising highquality prostate MRI, as this is of paramount importance especially when it comes to MRI-derived targeted biopsies used to detect clinically significant prostate cancer, since a patient with a negative scan and favourable prostate-specific antigen (PSA) kinetics should confidently avoid unnecessary immediate biopsy [13]. However, in order to be able to safely rule in and rule out prostate cancer, images with good spatial resolution, high signal-to-noise ratio and no artefacts are necessary.

The level of reproducibility found in our study compares favourably with that reported for other scoring systems of the prostate $[14,15]$ including PI-RADS [16-21], and similar results have been reported in the arterial hyperenhancement for the diagnosis of hepatocellular carcinoma using a 1-to-5 scoring system [22] and for the Bosniak classification of cystic renal masses [23].

In addition to this, Brembilla and colleagues have recently reported that interobserver studies in prostate MRI research should mirror clinical practice as closely as possible to increase the generalisability of the results [24].

At present, little is known about the effect of imaging quality on interobserver agreement [16] and a recent international consensus meeting has reiterated the importance of studies focusing on reporting MRI quality, as this is critical in studies evaluating prostate MRI performance and reproducibility [3].

The PI-RADS guidelines are well established [25] and, as such, have already been evaluated in studies that have
Table 4 PI-QUAL scores stratified in three different subgroups $(n=103)$ as assessed by each reader

\begin{tabular}{llllll}
\hline & & & & Total \\
\cline { 3 - 5 } & & & & & \\
\hline \multirow{2}{*}{ Reader 1 } & PI-QUAL 1-2 & 15 & PI-QUAL 3 & PI-QUAL 4-5 & \\
& PI-QUAL 3 & 4 & 38 & 1 & 18 \\
& PI-QUAL 4-5 & 0 & 8 & 1 & 43 \\
Total & & 19 & 48 & 34 & 42 \\
\hline
\end{tabular}

PI-QUAL, Prostate Imaging Quality 
Table 5 Parameters included in the visual assessment of the PI-QUAL scoring sheet in the 16 cases of disagreement

\section{Reader $1 \quad$ Reader 2}

\section{T2-WI}

Capsule clearly delineated

Seminal vesicles clearly delineated

Ejaculatory ducts clearly delineated

Neurovascular bundles clearly delineated

Sphincter muscle clearly delineated

Absence of artefacts

DWI

Adequate ADC map

Absence of artefacts

DCE

Capsular vessels clearly delineated

Vessels in the Alcock's canal clearly delineated

Absence of artefacts

Absented

T2-WI, T2-weighted imaging; $D W I$, diffusion-weighted imaging; $A D C$, apparent diffusion coefficient; $D C E$, dynamic contrast enhanced demonstrated their generalisability and reliability. The PIQUAL score, on the other hand, had been more recently developed and as such it is important to determine the reproducibility of the system [26].

If we have a closer look at Table 5 , we can see that the highest levels of disagreement in terms of visual assessment on T2-WI and DCE corresponded to the delineation of specific anatomic landmarks, such as the ejaculatory ducts, the neurovascular bundles and the urethral sphincter muscles on T2-WI along with the vessels on DCE, while higher agreement was observed as far as the prostatic capsule and seminal vesicles on T2-WI are concerned. This could be explained by the fact that some structures (e.g. the ejaculatory ducts and the urethral sphincter muscles) are more difficult to be identified and one of the differences that emerged during the postscoring consensus meeting was that the senior consultant ra-

Fig. 4 Six examples in which the two readers showed disagreement. T2-weighted imaging: in a the disagreement pertained to the delineation of the prostatic capsule while in $\mathbf{b}$ of the ejaculatory ducts (arrows). The final consensus was that the two scans were of suboptimal image quality. Diffusion-weighted imaging: in $\mathbf{d}$ the disagreement pertained to the adequacy of the ADC map (the corresponding high $\mathrm{b}$ sequence is provided in $\mathbf{c}$ for the sake of completeness). The final consensus was that the ADC map was not of adequate diagnostic quality. Dynamiccontrast enhanced sequences: the arrowheads in $\mathbf{e}$ and $\mathbf{f}$ are indicating the vessels in the Alcock's (or pudendal) canal in two different patients. One reader scored both scans as of suboptimal quality but after consensus meeting the readers agreed that the Alcock's (or pudendal) canal was clearly delineated in the two scans
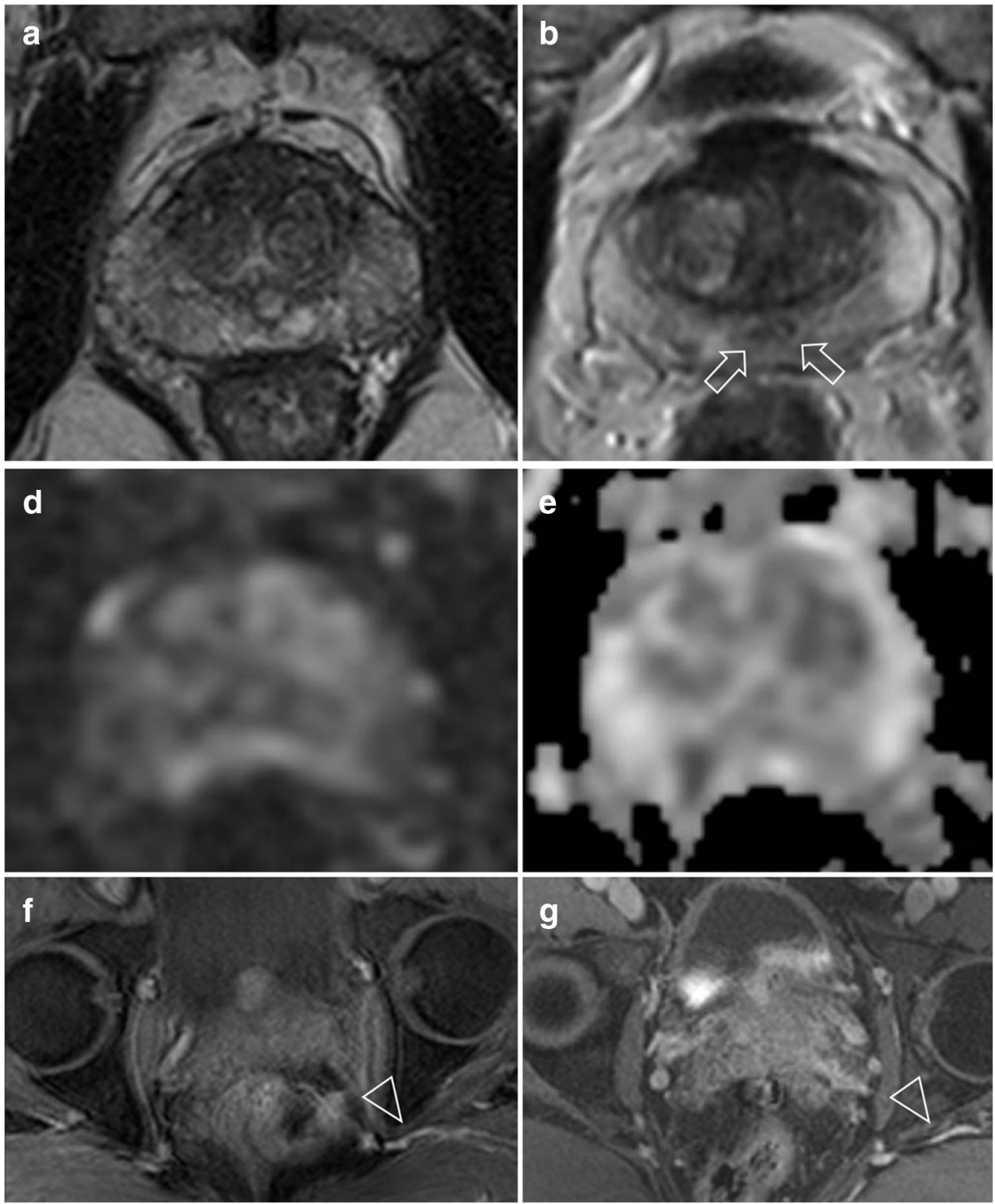

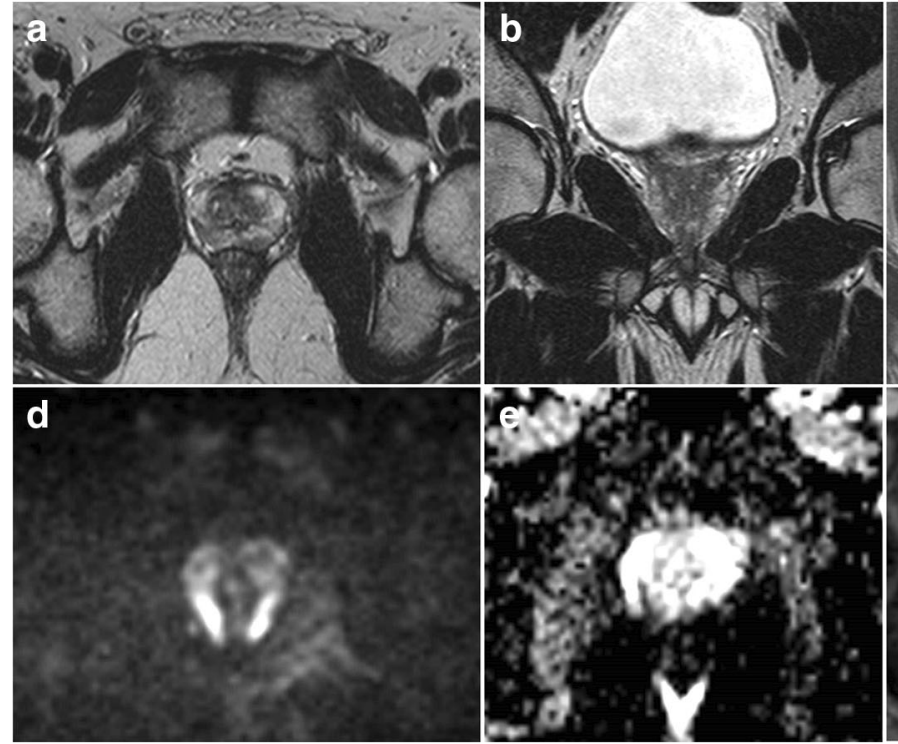

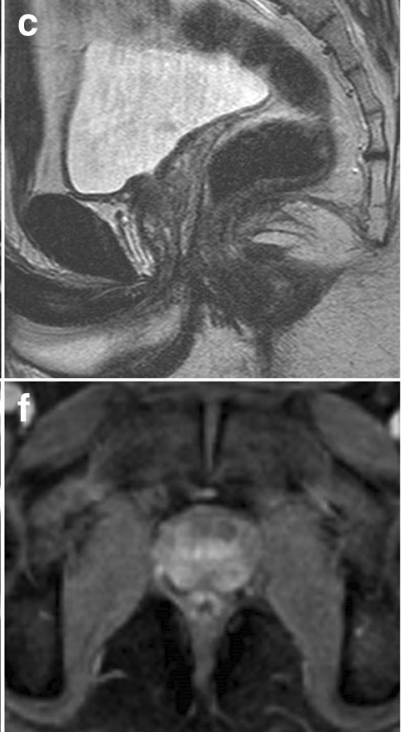

in-plane resolution and dynamic contrast-enhanced (DCE) sequences (f) showed suboptimal in-plane resolution and capsular vessels not clearly demarcated. The PI-QUAL score for both readers was 2 (i.e., only one MR sequence is of acceptable diagnostic quality). Final pathology confirmed bilateral Gleason $3+4$ (pT2c), with an overall tumour volume of $1.7 \mathrm{cc}$ and positive intraprostatic margins on both sides
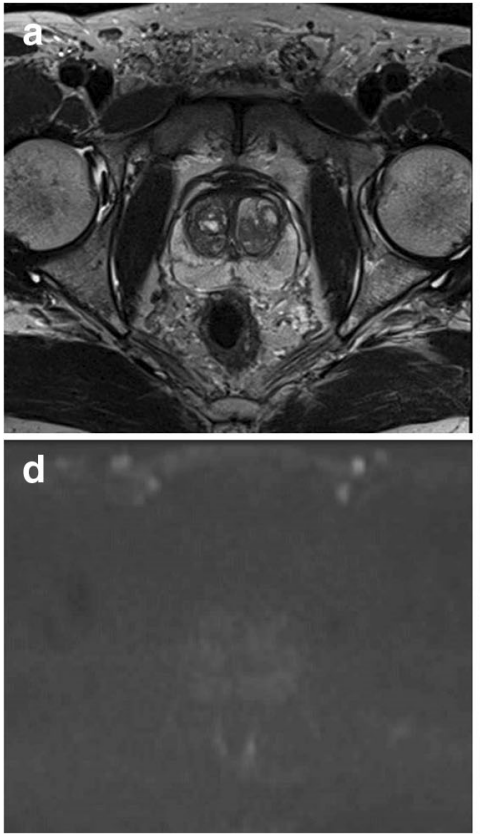

Fig. 6 Images in a 50-year-old patient scanned on a 3-T MR system with a presenting PSA of $5 \mathrm{ng} / \mathrm{ml}$ and a prostate volume of $80 \mathrm{cc}$ (PSA density: $0.06 \mathrm{ng} / \mathrm{ml} / \mathrm{ml}$ ), and bilateral Gleason $3+4$ at biopsy. Axial (a) and coronal (b) T2-weighted images were judged of adequate diagnostic quality, and the arrowheads in (b) indicate the internal urethral sphincter clearly demarcated. The $b=1000 \mathrm{~s} / \mathrm{mm}^{2}$ (c) and the high $b$ sequences $\left(b=1400 \mathrm{~s} / \mathrm{mm}^{2}\right)(\mathbf{d})$ along with the apparent diffusion coefficient (e) map from diffusion-weighted imaging were of acceptable diagnostic quality but the in-plane resolution of (d) and (e) was deemed suboptimal
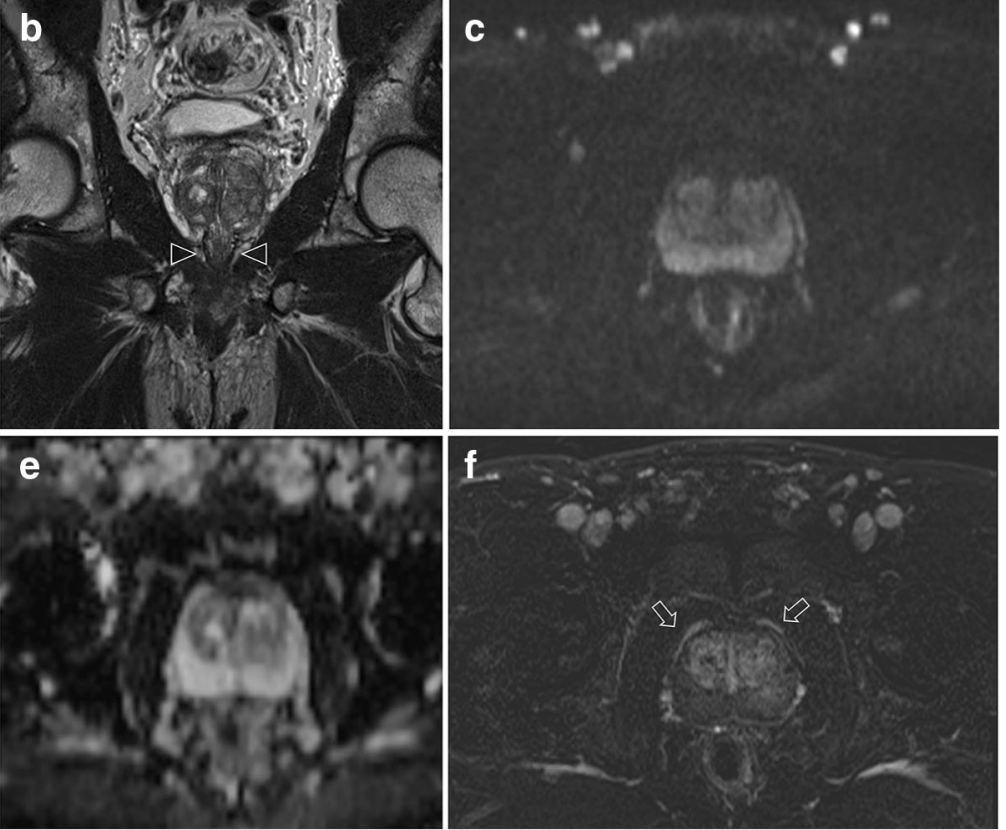

by both readers. The subtracted dynamic contrast-enhanced sequences (f) were of adequate diagnostic quality (the arrows indicate the capsular vessels clearly demarcated) but the temporal resolution was $20 \mathrm{~s}$ (i.e., above the threshold of $10 \mathrm{~s}$ as per PI-RADS v.2.0 guidelines). The PIQUAL score for both readers was 4 (i.e., two or more mpMRI sequences are independently of diagnostic quality). Final pathology confirmed bilateral organ-confined Gleason $3+4(\mathrm{pT} 2 \mathrm{c})$, with an overall tumour volume of $0.7 \mathrm{cc}$ 
diologist in prostate MRI reading used both the axial and coronal acquisitions to judge the visibility of these structures, while the other radiologist used only the axial T2-WI [27]. Also, the visual assessment of DCE images is more difficult compared to T2-WI, as multiple dynamic time-points need to be reviewed without a clear temporal definition for what constitutes early enhancement (e.g. a possible solution could be to use the time point when the adenoma in the transitional zone just starts to enhance, which should occur before any peripheral zone enhancement if the temporal resolution is adequate).

As far as DWI is concerned, the disagreement was substantial when assessing the adequacy of the apparent diffusion coefficient (ADC) map. This is an interesting point, as visual assessment is often used as the primary method to assess the quality of DWI [28] and this could be strengthened by the extrapolation of standardised ADC values from different institutions and MRI scanners or by using built-in phantoms on each scanner.

Interestingly, in the 16 cases reported in Table 5, the two readers had substantial agreement in the evaluation of the absence of artefacts on all sequences, in particular on T2-WI and DCE.

A key feature of our study is its multi-centre setting, which reflects the heterogeneity of prostate MRI conduct across different institutions and is representative of patients undergoing prostate MRI during routine clinical practice, thereby reinforcing the generalisability of our findings. Moreover, both readers interpreted the full examinations on imaging workstations rather than screen captures, ensuring the most accurate assessment for each MRI study.

Our results suggest that the PI-QUAL score can be applied with reasonable consistency by radiologists familiar with prostate MRI on the basis of a clear understanding of the lexicon provided in the scoring sheet [29]. However, it needs to be stressed that only genitourinary radiologists with appropriate training in prostate MRI should assess the quality of the scans [30]. The findings of our study may be also useful for guiding the future iteration of the PI-QUAL score, which should focus on the features that have the greatest objective reproducibility and that should receive more emphasis in the future versions.

Also, a more objective definition of some less reproducible features such as the 'reader-estimated' criteria (e.g. what constitutes 'adequacy' of the ADC map) will need to be addressed in the next version of PI-QUAL.

Our study has some limitations. Firstly, both readers assessing the PI-QUAL score worked at the same centre and the junior consultant radiologist had been previously trained by the senior consultant radiologist; thus, they may have had the tendency to approach cases similarly. It should be also noted that the PI-QUAL score has been only recently published [5] and there is currently no literature on its application in a clinical setting.
Another limitation of the present study is that we did not investigate the correlation of PI-QUAL with radical prostatectomy. Although we know that the use of PIQUAL has clinical implications (i.e., ruling in and ruling out clinically significant prostate cancer), the aim of this specific study was to test the interobserver reproducibility of the PI-QUAL score and not to evaluate the correlation between image quality, lesion conspicuity on mpMRI and histology. Though we did not test the inter-reader variability in ruling in and ruling out clinically significant prostate cancer, we can anticipate that other work is already underway to investigate PIQUAL score and diagnostic accuracy with pathology as reference standard. It should be also noted that the population included in this study consisted of patients who were candidates for radical prostatectomy, so there could have been a bias towards those studies with MR-visible lesions and higher PI-RADS scores.

As previously stated in the original publication [5], the PIQUAL score will be refined in the future (e.g. an international group is currently working on the next version of PI-QUAL in order to see if this scoring system should be still based on a 5point scale or simplified into a 3-point scale) and we believe that the findings of our study may be useful for guiding the future iteration of this scoring system. Thus, it is envisaged that the interobserver agreement will continue to improve over time with the new iterations of the PI-QUAL score.

In conclusion, the findings of our study support the reliability of the PI-QUAL score for the assessment of prostate MRI quality. However, it is anticipated that the scoring system will need to undergo further refinements.

Supplementary Information The online version contains supplementary material available at https://doi.org/10.1007/s00330-021-08169-1.

Acknowledgements F.G. is a recipient of the 2020 Young Investigator Award funded by the Prostate Cancer Foundation / CRIS Cancer Foundation. ED is funded by NIHR RfPB and JP Moulton Foundation. VK is an Academic Clinical Lecturer funded by the UK National Institute for Health Research (NIHR). SP receives research support from the United Kingdom's National Institute of Health Research (NIHR) UCLH/UCL Biomedical Research Centre. AK is supported by the UCLH/UCL Biomedical Research Centre. ME is a United Kingdom National Institute of Health Research (NIHR) Senior Investigator and receives research support from the UCLH/UCL NIHR Biomedical Research Centre. CMM is supported by the UKNIHR, Movember, PCUK and the EAU Research Foundation. The NeuroSAFE PROOF team would like to thank the participants, principal investigators, research nurses, clinicians involved in providing care, regional cancer service coordinators, data managers, and other site staff responsible for setting up, recruiting participants, and collecting data for the trial. They would like to thank members of the Data Monitoring Committee and members of Trial Steering Committee. 
Funding information The NeuroSAFE PROOF feasibility trial was funded by the National Institute for Health Research (NIHR) Research for Patient Benefit (RfPB) programme (PB-PG- 1216-20013) and The Rosetrees Foundation.

\section{Declarations}

Guarantor The scientific guarantor of this publication is Francesco Giganti.

Conflict of interest The authors of this manuscript declare no relationships with any companies, whose products or services may be related to the subject matter of the article.

Statistics and biometry No complex statistical methods were necessary for this paper.

Informed consent Written informed consent was obtained from all subjects (patients) in this study.

Ethical approval Institutional Review Board approval was obtained.

\author{
Methodology \\ - retrospective \\ - randomised controlled trial \\ - multi-centre study
}

Open Access This article is licensed under a Creative Commons Attribution 4.0 International License, which permits use, sharing, adaptation, distribution and reproduction in any medium or format, as long as you give appropriate credit to the original author(s) and the source, provide a link to the Creative Commons licence, and indicate if changes were made. The images or other third party material in this article are included in the article's Creative Commons licence, unless indicated otherwise in a credit line to the material. If material is not included in the article's Creative Commons licence and your intended use is not permitted by statutory regulation or exceeds the permitted use, you will need to obtain permission directly from the copyright holder. To view a copy of this licence, visit http://creativecommons.org/licenses/by/4.0/.

\section{References}

1. Barentsz JO, Richenberg J, Clements R et al (2012) ESUR prostate MR guidelines 2012. Eur Radiol 22(4):746-757

2. Giganti F, Rosenkrantz AB, Villeirs GM et al (2019) The evolution of MRI of the prostate: the past, the present, and the future. AJR Am J Roentgenol 213(2):384-396

3. De Rooij M, Israël B, Tummers M et al (2020) ESUR / ESUI consensus statements on multi-parametric MRI for the detection of clinically significant prostate cancer: quality requirements for image acquisition, interpretation and radiologists' training. Eur Radiol 30(10):5404-5416

4. Brizmohun Appayya M, Adshead J, Ahmed H et al (2018) National Implementation of multi-parametric MRI for prostate cancer detection - recommendations from a UK consensus meeting. BJU Int 122(1): 13-25

5. Giganti F, Allen C, Emberton M, Moore CM, Kasivisvanathan V (2020) Prostate Imaging Quality (PI-QUAL): a new quality control scoring system for multiparametric magnetic resonance imaging of the prostate from the PRECISION trial. Eur Urol Oncol 3(5):615619
6. Kasivisvanathan V, Rannikko AS, Borghi M et al (2018) MRItargeted or standard biopsy for prostate-cancer diagnosis. N Engl J Med 378(19):1767-1777

7. Dinneen E, Haider A, Allen C et al (2019) NeuroSAFE robotassisted laparoscopic prostatectomy versus standard robot- assisted laparoscopic prostatectomy for men with localised prostate cancer (NeuroSAFE PROOF): protocol for a randomised controlled feasibility study. BMJ Open 9(6):e028132

8. Weinreb JC, Barentsz JO, Choyke PL et al (2016) PI-RADS Prostate Imaging - Reporting and Data System: 2015, Version 2. Eur Urol 69(1):16-40

9. Turkbey B, Rosenkrantz AB, Haider MA et al (2019) Prostate Imaging Reporting and Data System Version 2.1: 2019 Update of Prostate Imaging Reporting and Data System Version 2. Eur Urol 0232:1-12

10. Feinstein AR, Cicchetti DV (1990) High agreement but low kappa: I. the problems of two paradoxes. J Clin Epidemiol 43(6):543-549

11. Shankar V, Bangdiwala SI (2014) Observer agreement paradoxes in $2 \times 2$ tables: comparison of agreement measures. BMC Med Res Methodol 14:100

12. Lantz CA, Nebenzahl E (1996) Behavior and interpretation of the kappa statistic: resolution of the two paradoxes. J Clin Epidemiol 49(4):431-434

13. Panebianco V, Barchetti G, Simone G et al (2018) Negative multiparametric magnetic resonance imaging for prostate cancer: what's next? Eur Urol 74(1):48-54

14. Renard-Penna R, Mozer P, Cornud F et al (2015) Prostate Imaging Reporting and Data System and Likert scoring system: multiparametric MR imaging validation study to screen patients for initial biopsy. Radiology 275(2):458-468

15. Giganti F, Pecoraro M, Stavrinides V et al (2020) Interobserver reproducibility of the PRECISE scoring system for prostate MRI on active surveillance: results from a two-centre pilot study. Eur Radiol 30(4):2082-2090

16. Park KJ, Choi SH, Lee JS, Kim JK, Kim M (2020) Interreader Agreement with Prostate Imaging Reporting and Data System Version 2 for prostate cancer detection: a systematic review and meta-analysis. J Urol 204(4):661-670

17. Rosenkrantz AB, Kim S, Lim RP et al (2013) Prostate cancer localization using multiparametric MR imaging: comparison of Prostate Imaging Reporting and Data System (PI-RADS) and Likert scales. Radiology 269(2):482-492

18. Schimmöller L, Quentin M, Arsov C, Lanzman RS (2013) Interreader agreement of the ESUR score for prostate MRI using in-bore MRI-guided biopsies as the reference standard. Eur Radiol 23(11): 3185-3190

19. Rosenkrantz AB, Ginocchio LA, Cornfeld D et al (2016) Interobserver Reproducibility of the PI-RADS Version 2 Lexicon: a multicenter study of six experienced prostate radiologists. Radiology 280(3):793-804

20. Vaché T, Bratan F, Mège-Lechevallier F, Roche S, Rabilloud M, Rouvière $\mathrm{O}$ (2014) Characterization of prostate lesions as benign or malignant at multiparametric MR imaging: comparison of three scoring systems in patients treated with radical prostatectomy. Radiology 272(2):446-455

21. Brembilla G, Dell ' Oglio P, Stabile A et al (2020) Interreader variability in prostate MRI reporting using Prostate Imaging Reporting and Data System version 2.1. Eur Radiol 30(6):33833392

22. Davenport MS, Khalatbari S, Liu PSC et al (2014) Repeatability of diagnostic features and scoring systems for hepatocellular carcinoma by using MR imaging. Radiology 272(1):132-142

23. Bai X, Sun SM, Xu W et al (2020) MRI-based Bosniak Classification of Cystic Renal Masses, Version 2019: interobserver 
agreement, impact of readers' experience, and diagnostic performance. Radiology 297(3):597-605

24. Brembilla G, Takwoingi Y, Kasivisvanathan V (2021) Tackling interobserver variability in multiparametric magnetic resonance imaging (MRI): is MRI even better than we think for prostate cancer diagnosis? Eur Urol 79(1):8-10

25. Padhani AR, Barentsz J, Villeirs G et al (2019) PI-RADS Steering Committee: The PI-RADS multiparametric MRI and MRI-directed biopsy pathway. Radiology 292(2):464-474

26. Stabile A, Giganti F, Kasivisvanathan V et al (2020) Factors influencing variability in the performance of multiparametric magnetic resonance imaging in detecting clinically significant prostate cancer: a systematic literature review. Eur Urol Oncol 3(2):145167

27. Bonekamp D, Jacobs MA, El-Khouli R, Stoianovici D, Macura KJ (2011) Advancements in MR imaging of the prostate: from diagnosis to interventions. Radiographics 31(3):677-703
28. Kwon MR, Kim C, Kim JH (2017) PI-RADS version 2: evaluation of diffusion-weighted imaging interpretation between $\mathrm{b}=1000$ and $\mathrm{b}=1500 \mathrm{~s} \mathrm{~mm}^{-2}$. Br J Radiol 90(1079):20170438

29. Giganti F, Kirkham A, Kasivisvanathan V et al (2021) Understanding PI-QUAL for prostate MRI quality: a practical primer for radiologists. Insights Imaging 12(1):59

30. Padhani AR, Schoots IG, Turkbey B, Giannarini G, Barentsz JO (2020) A multifaceted approach to quality in the MRI-directed biopsy pathway for prostate cancer diagnosis. Eur Radiol. https://doi. org/10.1007/s00330-020-07527-9

Publisher's note Springer Nature remains neutral with regard to jurisdictional claims in published maps and institutional affiliations. 